Check for updates

Cite this: RSC Adv., 2018, 8, 39703

Received 19th October 2018

Accepted 22nd November 2018

DOI: $10.1039 / c 8 r a 08669 a$

rsc.li/rsc-advances

\section{Tungsten disulfide nanosheets supported poly(xanthurenic acid) as a signal transduction interface for electrochemical genosensing applications}

\begin{abstract}
Jimin Yang, $\mathbb{D} \dagger$ Xuesong Yin, $\uparrow$ Min Xia and Wei Zhang $(\mathbb{D}$ *
Tungsten disulfide $\left(W_{2}\right)$ nanosheets supported poly(xanthurenic acid) (PXa) was used as the signal transduction interface for electrochemical genosensing. The $\mathrm{WS}_{2}$ nanosheets were obtained from bulk $W_{2}$ using a simple ultrasonic method. Due to the unique physical adsorption of Xa monomers to $W_{2}$, the electropolymerization efficiency was greatly improved, accompanied with an increased electrochemical response of PXa. The obtained $\mathrm{PXa} / \mathrm{WS}_{2}$ nanocomposite not only served as a substrate for DNA immobilization but also reflected the electrochemical transduction originating from DNA immobilization and hybridization without any other indicators or complicated labelling steps. Owing to the presence of abundant carboxyl groups, the probe ssDNA was covalently attached on the carboxyl-terminated $\mathrm{PXa}_{\mathrm{W}} \mathrm{WS}_{2}$ nanocomposite through the free amines of DNA sequences based on the 1-ethyl-3-(3-dimethylaminopropyl) carbodiimide and $\mathrm{N}$-hydrosulfosuccinimide crosslinking reaction. The covalently immobilized probe ssDNA could selectively hybridize with its target DNA to form dsDNA on the surface of the PXa/WS 2 nanocomposite. This developed biosensor achieved a satisfactory detection limit down to $1.6 \times 10^{-16} \mathrm{~mol} \mathrm{L^{-1 }}$ and a dynamic range of $1.0 \times$ $10^{-15}$ to $1.0 \times 10^{-11} \mathrm{~mol} \mathrm{~L}^{-1}$ for detection of circulating tumor DNA related to gastric carcinoma. Selectivity of the biosensor has been investigated in presence of non-complementary, onemismatched and two-mismatched DNA sequences.
\end{abstract}

\section{Introduction}

In recent years, much attention has been focused on utilizing circulating cell-free DNA, specifically the tumor-derived circulating cell-free DNA (circulating tumor DNA, ctDNA) isolated from peripheral blood, as a biomarker of disease status in metastatic cancer patients. In cancer patients, the high cell turnover rate of tumors results in the release of ctDNA. Numerous studies have shown that the ctDNA carries genetic information from the entire tumor genome and can therefore provide insight into clonal heterogeneity and evolution of all solid cancers present at any one time..$^{1-3}$ Thus, the presence of ctDNA in the blood provides information via a minimally invasive liquid biopsy, eliminating the morbidity associated with serial sampling of tumors for monitoring patients with advanced solid cancers. ${ }^{4-7}$ Utilizing the ctDNA as the liquid biopsy has significant potential to pave the way toward a better understanding of cancer at the molecular level and improve patient outcomes in the future. The

School of Chemistry and Chemical Engineering, Linyi University, Linyi 276005, China. E-mail: zhangweiqust@126.com; Fax:+86-539-7258620; Tel: +86-539-7258620

$\dagger$ These two authors contributed equally to this work. traditional methods for analyzing the ctDNA in the blood mainly include the polymerase chain reaction and DNA sequencing. Although these technologies have led to significant contributions to the ctDNA detection, further application is still restricted by the complicated sample preparation and the interference caused from the constituents of the biological environment. ${ }^{8,9}$

Layered two-dimensional (2D) transition metal dichalcogenides (TMDCs) with single or few atomic layers have attracted immense interest due to their special structures with high specific surface area and unusual electronic properties. ${ }^{10-12}$ As one of the newly emerging 2D TMDCs, layered tungsten disulfide $\left(\mathrm{WS}_{2}\right)$ composed of S-W-S sandwiches in a trigonal prismatic coordination is a typical example of inorganic analogues of graphene, ${ }^{13,14}$ which has shown promising prospect in the field of electrocatalysts, batteries, supercapacitors, and so on. ${ }^{15-17}$ Nevertheless, less attention has been paid to its application as the electrode material for electrochemical sensing because the electronic conductivity of $\mathrm{WS}_{2}$ is still relatively low, similar to the most transition metal oxides. To overcome this problem, hybrid materials that incorporated $\mathrm{WS}_{2}$ with high electronic conductive materials seem to be imperative. 
As a novel conducting polymer, poly(xanthurenic acid) (PXa), is of much interest due to its several advantages including high redox activity and low toxicity, which has been used for determination of biomolecules. ${ }^{18-20}$ In this work, Xa was electropolymerized on the pre-obtained $\mathrm{WS}_{2}$ nanosheets substrate to obtain a novel polymer nanocomposite, which was adopted as an ideal interface for electrochemical determination of the PIK3CA gene. The PIK3CA gene existing in the peripheral blood of patients with gastric carcinoma is a kind of ctDNA. The probe ssDNA was covalently linked to the PXa/ $\mathrm{WS}_{2}$ nanocomposite via the free amines of ssDNA and the carboxyl groups of PXa, which caused a "signal-off" model with decreased current, ascribed to flexible probe ssDNA strands covering the surface and blocking the electron transfer channel between the electrode interface and the solution. ${ }^{21}$ After hybridization with the complementary sequence, the conformational changes and the different properties between sSDNA and dsDNA could affect the redox kinetics of the functional nanocomposite, ${ }^{22,23}$ followed by an increase ("signal-on") of the redox current. The self-signal changes induced by DNA immobilization and hybridization could be sensitively recognized via cyclic voltammetry (CV) and electrochemical impedance spectroscopy (EIS). To the best of our knowledge, the integration of an unconventional conductive polymer (PXa) and $\mathrm{WS}_{2}$ nanosheets as the sensing interface for the direct electrochemical detection of DNA hybridization via self-signal changes has not yet been reported.

\section{Experimental}

\subsection{Apparatus and materials}

The electrochemical measurements were performed on a CHI660E electrochemical workstation (Shanghai $\mathrm{CH}$ Instrument Company, China). A conventional three-electrode system was used with carbon paste electrode (CPE, $\varnothing=3$ $\mathrm{mm}$ ) or the modified CPE as the working electrode, a saturated calomel electrode (SCE) as the reference electrode and a Pt wire as the counter electrode. Transmission electron microscopy (TEM) was carried out on a JEM-2100 machine (JEOL, Japan).

Graphite powder (spectral pure, diameter about $30 \mu \mathrm{m}$ ) was purchased from Shanghai Colloid Chemical Plant (China). The bulk $\mathrm{WS}_{2}$ was obtained from Shanghai Aladdin Biological Technology Corporation (China). Xa was acquired from Acros Organics (Belgium). 1-Ethyl-3-(3-dimethylaminopropyl) carbodiimide (EDC) and $N$-hydrosulfosuccinimide (NHS) were obtained from Sigma (USA). $N, N$-Dimethylformamide (DMF) and phosphate-buffered saline (PBS) solution were provided by Shanghai Reagent Company (China). All the chemicals were of analytical grade and used as received.

The DNA oligonucleotides of the PIK3CA gene related to gastric carcinoma were synthesized by Shanghai Sangon Bioengineering Limited Company (China). Their base sequences were listed below:

Probe DNA (ssDNA): $5^{\prime}-\mathrm{NH}_{2}-\mathrm{AGT}$ GAT TTT AGA GAG-3';

Complementary DNA (cDNA): $5^{\prime}$-CTC TCT AAA ATC ACT-3'; One-base mismatched DNA: $5^{\prime}$-CTC TCT AGA ATC ACT-3';
Two-base mismatched DNA: $5^{\prime}$-CTC TCT Ag A ATG ACT-3'; Noncomplementary DNA (ncDNA): 5'-TAC TCC GCG CTA ACG-3' .

\subsection{Electrochemical fabrication of $\mathrm{PXa} / \mathrm{WS}_{2} / \mathrm{CPE}$}

The CPE was fabricated as reported previously. ${ }^{24}$ The $\mathrm{WS}_{2}$ nanosheets were prepared by a simple ultrasonic exfoliation method. Briefly, bulk $\mathrm{WS}_{2}(10 \mathrm{mg})$ was transferred into DMF (10 $\mathrm{mL}$ ) followed by ultrasonication for $6 \mathrm{~h}$, and then a homogenous suspension was obtained with the concentration of $1.0 \mathrm{mg}$ $\mathrm{mL}^{-1}$. In order to obtain a smooth surface, the surface of CPE was pre-treated by polishing with a weighing paper. $10 \mu \mathrm{L}$ of the suspension was dripped on the pre-treated surface of $\mathrm{CPE}$ uniformly to obtain the $\mathrm{WS}_{2} / \mathrm{CPE}$.

The electropolymerization of PXa was accomplished by immersing the pre-obtained $\mathrm{WS}_{2} / \mathrm{CPE}$ in $0.3 \mathrm{~mol} \mathrm{~L}^{-1} \mathrm{PBS}(\mathrm{pH}$ 5.5) containing $400 \mu \mathrm{mol} \mathrm{L}{ }^{-1} \mathrm{Xa}$ monomers and then cycling the potential between $1.2 \mathrm{~V}$ and $-0.6 \mathrm{~V}$ with a scan rate of $50 \mathrm{mV}$ $\mathrm{s}^{-1}$ for 15 cycles, and the obtained electrode was denoted as $\mathrm{PXa} / \mathrm{WS}_{2} / \mathrm{CPE}$. For comparison, the PXa/CPE was also fabricated under the similar procedure.

\subsection{Immobilization and hybridization of DNA}

The ssDNA probes were covalently attached on the PXa/ $/ \mathrm{WS}_{2} /$ CPE surface through the free amines of ssDNA probes by using the EDC/NHS cross-linking reaction. The terminal carboxyl groups of the $\mathrm{PXa} / \mathrm{WS}_{2}$ nanocomposite were activated by immersion in $0.3 \mathrm{~mol} \mathrm{~L}^{-1} \mathrm{PBS}$ ( $\mathrm{pH} 7.0$ ) containing $2.0 \mathrm{mmol} \mathrm{L}^{-1}$ EDC and $5.0 \mathrm{mmol} \mathrm{L}^{-1} \mathrm{NHS}$ for $1 \mathrm{~h}$. The linker/PXa/WS $/ \mathrm{CPE}$ was rinsed with PBS ( $\mathrm{pH} 7.0$ ) to wash off the redundant EDC and NHS. $10 \mu \mathrm{L}$ of PBS $(\mathrm{pH}$ 7.0) containing ssDNA probes $(1.0 \times$ $10^{-11} \mathrm{~mol} \mathrm{~L}^{-1}$ ) was then pipetted onto the chemically modified electrode and air-dried, rinsed with ultrapure water to remove the unimmobilized ssDNA, and this probe-captured electrode was denoted as SSDNA/PXa/ $/ \mathrm{WS}_{2} / \mathrm{CPE}$.

Afterwards, $10 \mu \mathrm{L}$ of hybridization solution (PBS, $\mathrm{pH} 7.0$ ) containing cDNA was pipetted onto the probe-modified electrode, followed by thoroughly washed with ultrapure water to remove the unhybridized cDNA. The same procedures as mentioned above were applied to the probe-modified electrode for hybridization with ncDNA $\left(1.0 \times 10^{-11} \mathrm{~mol} \mathrm{~L}^{-1}\right)$, one-base mismatched DNA $\left(1.0 \times 10^{-11} \mathrm{~mol} \mathrm{~L}^{-1}\right)$ and two-base mismatched DNA $\left(1.0 \times 10^{-11} \mathrm{~mol} \mathrm{~L}^{-1}\right)$.

\subsection{Electrochemical measurements}

The CV measurements were carried out in $0.3 \mathrm{~mol} \mathrm{~L}^{-1} \mathrm{PBS}(\mathrm{pH}$ 7.0) from $0.3 \mathrm{~V}$ to $-0.4 \mathrm{~V}$ with a scan rate of $100 \mathrm{mV} \mathrm{s}^{-1}$. The EIS measurements were performed in $0.3 \mathrm{~mol} \mathrm{~L}^{-1} \mathrm{PBS}(\mathrm{pH} 7.0)$ with the AC voltage amplitude of $5 \mathrm{mV}$, and the voltage frequencies ranged from $10^{5} \mathrm{~Hz}$ to $1 \mathrm{~Hz}$.

The reported result for every electrode in this assay was the mean of three independent measurements. During the whole experiments, the solution was continuously bubbled with nitrogen for removing the oxygen. Unless specifically noted, all the experiments were carried out at room temperature. 


\section{Results and discussion}

\subsection{TEM characterization}

TEM were used to characterize the morphology of the $\mathrm{PXa} / \mathrm{WS}_{2}$ composite. The representative TEM images of the $\mathrm{WS}_{2}, \mathrm{PXa} / \mathrm{WS}_{2}$ composite and PXa were displayed in Fig. 1. Apparently, the $\mathrm{WS}_{2}$ (Fig. 1A) showed a nanosheet structure with smooth basal, and the nanosheets overlapped with each other and stacked on the electrode surface. After the polymerization of Xa, the nanosheet surface of $\mathrm{PXa} / \mathrm{WS}_{2}$ composite (Fig. 1B) was uneven and showed many swellings. It may be because PXa growing on the surface of $\mathrm{WS}_{2}$ formed relatively rough film. However, as shown in Fig. 1C, PXa formed on the bare CPE without $\mathrm{WS}_{2}$ exhibited an unsmooth morphology with many wrinkles.

\subsection{Electrochemical synthesis of the $\mathbf{P X a} / \mathrm{WS}_{2}$ nanocomposite}

As shown in Fig. 2A, a quasi-reversible redox (peaks a and b in Fig. 2A) revealing the formation of PXa appeared and increased with the increment of the CV cycles. The specific oxidation peak (peak c in Fig. 2A) could also be seen which indicated the oxidation and electropolymerization of Xa. Obviously, the specific peaks of $\mathrm{PXa} / \mathrm{WS}_{2} / \mathrm{CPE}$ (red line in Fig. 2A) were larger than those of PXa/CPE (blue line in Fig. 2A), which demonstrated that the $\mathrm{WS}_{2}$ nanosheets had a positive influence on the electropolymerization of Xa. This might be due to the unique physical adsorption of Xa monomers with the $\mathrm{WS}_{2}$ nanosheets, which could provide more nucleation sites and effectively improve the electropolymerization efficiency.

To further investigate the influence of $\mathrm{WS}_{2}$ nanosheets on the electrochemical polymerization, the electrochemical properties of different modified electrodes were compared by monitoring the self-redox signals of PXa in $0.3 \mathrm{~mol} \mathrm{~L}^{-1} \mathrm{PBS}(\mathrm{pH}$ 7.0), as displayed in Fig. 2B. No peaks were observed on the bare CPE (curve a). The voltammetric response of the PXa/CPE (curve b) consisted of a couple of redox peaks, which could be ascribed to the self-redox reactions of the electrodeposited PXa film that occur at the electrode surface and the redox process going from quinone structure to hydroquinone structure (inset in Fig. 2A). The current response of the $\mathrm{PXa} / \mathrm{WS}_{2} / \mathrm{CPE}$ (curve c) showed a much larger value compared with that of the PXa/CPE (curve b), demonstrating that the presence of $\mathrm{WS}_{2}$ nanosheets could dramatically enhance the electropolymerization efficient and resulted in an improved electrochemical self-redox response of PXa.

\subsection{Optimization of experimental conditions}

The amount of $\mathrm{WS}_{2}$ nanosheets could play a key role in the performance of the $\mathrm{PXa} / \mathrm{WS}_{2}$ nanocomposite film. With the increase of the amount of $\mathrm{WS}_{2}$ nanosheets, the electrochemical response of the $\mathrm{PXa} / \mathrm{WS}_{2}$ nanocomposite increased gradually, and the maximum was obtained at $10 \mu \mathrm{L}$ of $1.0 \mathrm{mg} \mathrm{mL}^{-1} \mathrm{WS}_{2}$ nanosheets. The low concentration of $\mathrm{WS}_{2}$ nanosheets will result in too thin $\mathrm{WS}_{2}$ film, so the few binding sites of thin $\mathrm{WS}_{2}$ film with Xa result in the weak physical adsorption between $\mathrm{WS}_{2}$ and $\mathrm{Xa}$. However, if the concentration of $\mathrm{WS}_{2}$ nanosheets was larger than $1.0 \mathrm{mg} \mathrm{mL}^{-1}$, the current response decreased, which may be attributed to much thicker $\mathrm{WS}_{2}$ sheets so that they stack together and affect the conductivity of the formed nanocomposite film. Therefore, $10 \mu \mathrm{L}$ of $1.0 \mathrm{mg} \mathrm{mL}^{-1} \mathrm{WS}_{2}$ nanosheets was selected for the electrode modification in experiments.

The electrochemical response of the $\mathrm{PXa} / \mathrm{WS}_{2}$ nanocomposite could also be obviously affected by the electrochemical preparation conditions, such as the electropolymerization cycles. The electrochemical signals of the $\mathrm{PXa} / \mathrm{WS}_{2}$ nanocomposite reached a maximum at the 15 th cycle, and then decreased with further scans. Therefore, a 15-cycle electropolymerization was chosen in experiments.

\subsection{Self-signal monitoring of DNA immobilization and hybridization}

The self-redox properties of the PXa layer were investigated to monitor the immobilization and hybridization of DNA on the $\mathrm{PXa} / \mathrm{WS}_{2}$ nanocomposite by $\mathrm{CV}$ in $0.3 \mathrm{~mol} \mathrm{~L}^{-1} \mathrm{PBS}(\mathrm{pH} 7.0)$ and shown in Fig. 3. Just as discussed above, the resulting $\mathrm{PXa} / \mathrm{WS}_{2} /$ CPE displayed fine electrochemical activities in a neutral
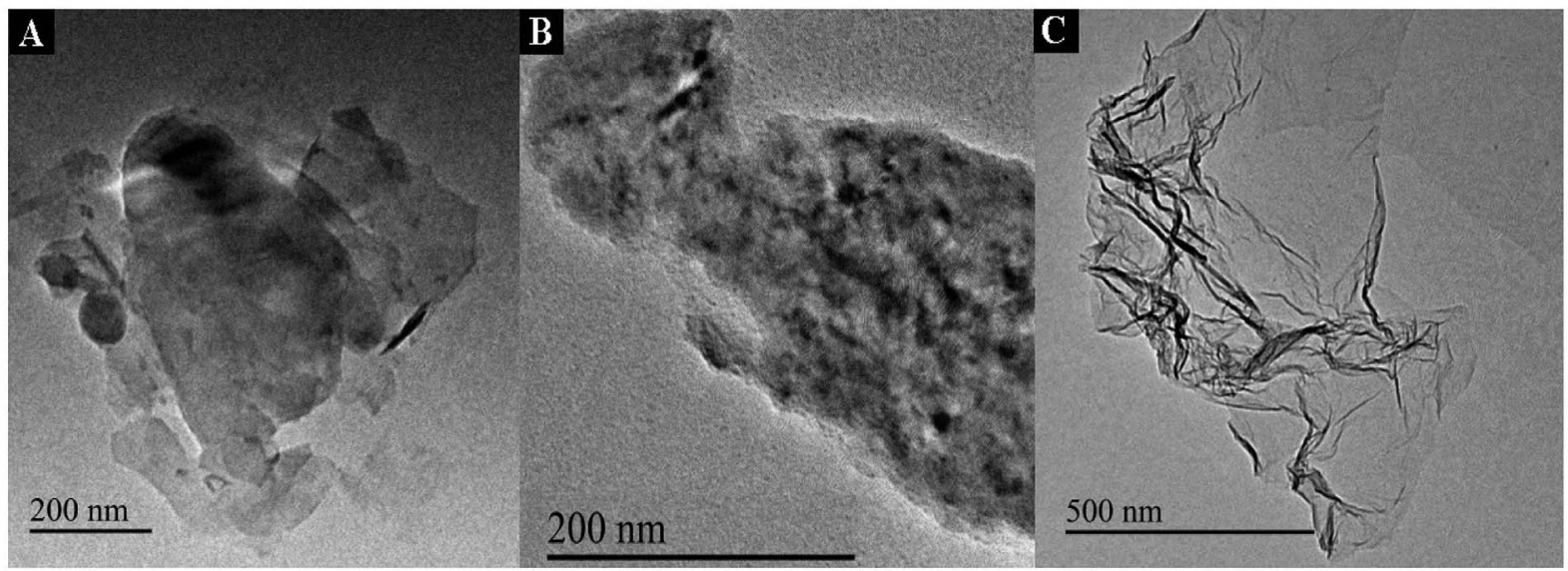

Fig. 1 TEM images of $W_{2}(A), P X a / W S_{2}(B)$ and PXa (C). 

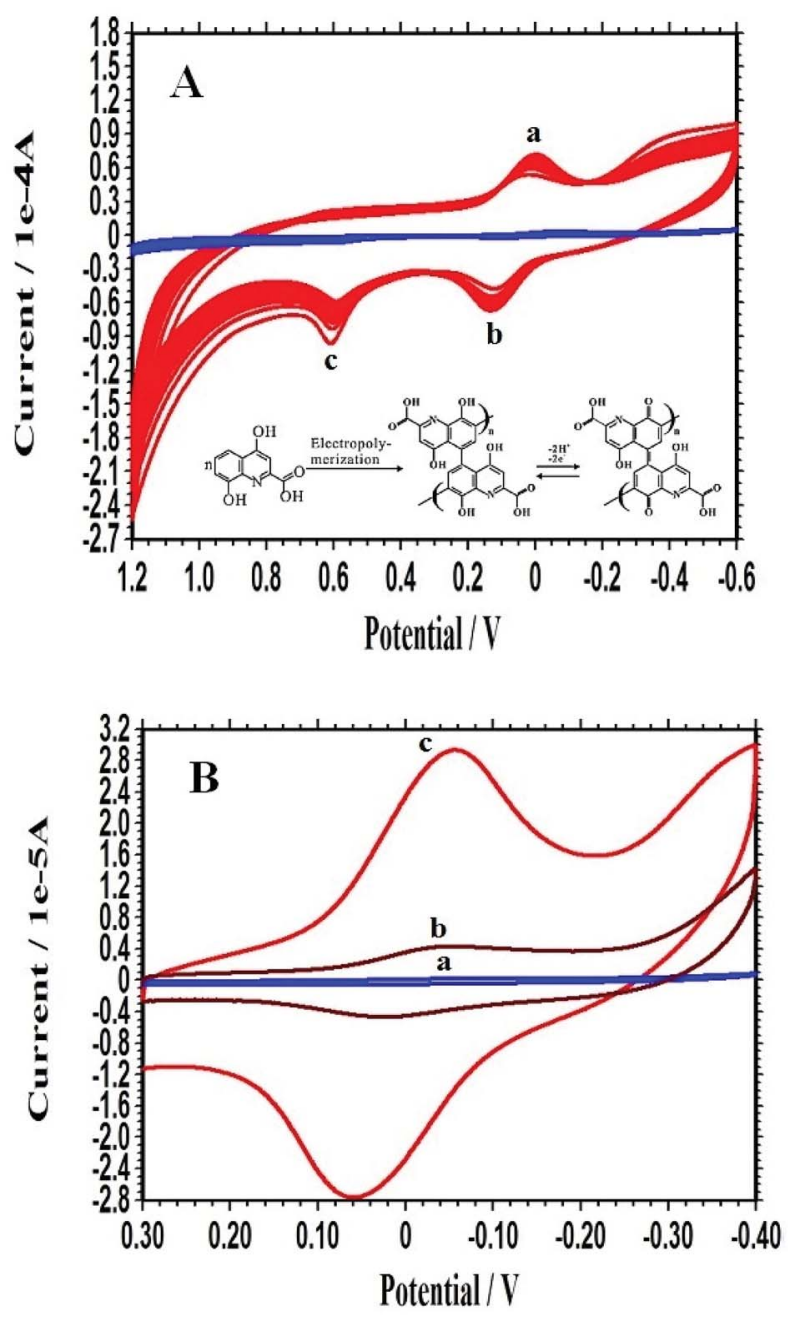

Fig. 2 (A) Electropolymerization process of $\mathrm{Xa}$ at different electrodes: $\mathrm{PXa} / \mathrm{CPE}$ (blue line) and $\mathrm{PXa} / \mathrm{WS} \mathrm{S}_{2} / \mathrm{CPE}$ (red line). (B) CVs of the bare CPE (a), $\mathrm{PXa} / \mathrm{CPE}$ (b) and $\mathrm{PXa} / \mathrm{WS}_{2} / \mathrm{CPE}$ (c) recorded in $0.3 \mathrm{~mol} \mathrm{~L}^{-1} \mathrm{PBS}(\mathrm{pH} 7.0)$.

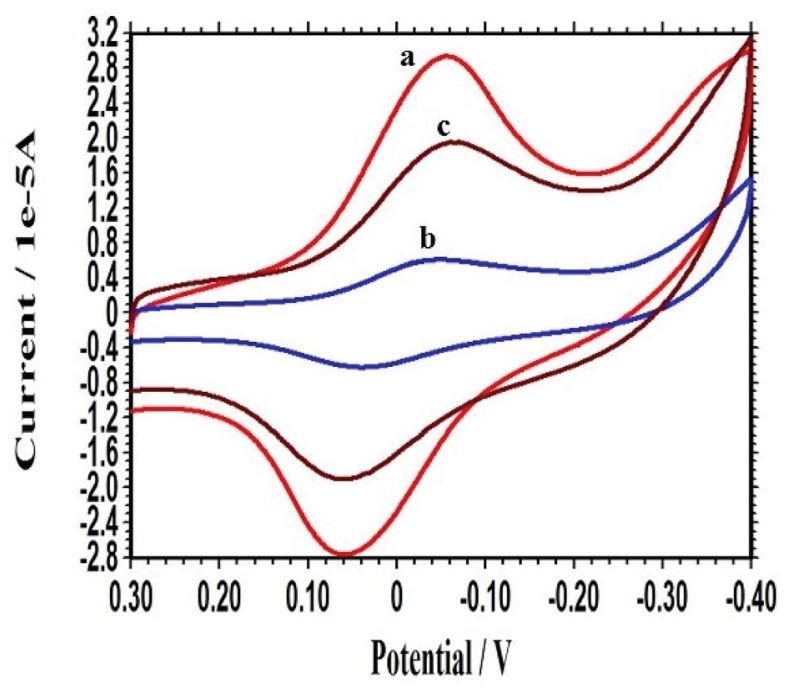

Fig. $3 \mathrm{CVs}$ of the $\mathrm{PXa} / \mathrm{WS}_{2} / \mathrm{CPE}$ (a), ssDNA/PXa/WS $/ \mathrm{CPE}$ (b) and dsDNA/PXa/WS $2 / C P E$ (c) recorded in $0.3 \mathrm{~mol} \mathrm{~L}^{-1} \mathrm{PBS}$ ( $\mathrm{pH} 7.0$ ). environment (curve a). Subsequently, after the $\mathrm{NH}_{2}$-ssDNA was captured through covalent bonding on the surface of the PXa/ $\mathrm{WS}_{2}$ nanocomposite, the self-redox signals of the ssDNA/PXa/ $\mathrm{WS}_{2} / \mathrm{CPE}$ decreased significantly (curve b). The probe ssDNA behaves as random coils and possesses the flexible traits, which may change the conformation and block the effective electron transfer along the PXa chains. ${ }^{21}$ Therefore, the response of the ssDNA/PXa/WS ${ }_{2}$ layer decreased ("signal-off"). However, when the ssDNA probes were hybridized with the cDNA, an increase of the CV signals (curve c) was observed compared with curve b. Interpretation of the current enhancement upon hybridization might be proposed on the basis of changes in the conformation of DNA. Indeed, the ssDNA behaves as random coils while the dsDNA leads to a more organized surface, through which counter ions along the double-helix structure could diffuse more freely. At the same time, ssDNA is a flexible molecule while dsDNA acts as a rigid rod, and hence the electron transfer rate along the double-helix structure is much faster than that along the single-chain structure. ${ }^{22,23}$ Therefore, the self-redox signals increased after the hybridization of ssDNA with its cDNA ("signal-on"). The above results demonstrated that the self-redox signal changes of the PXa layer could serve as a simple and efficient tool for direct DNA detection by CV without adding any labels to the ssDNA probes or target molecules and needing any outer indicators.

\subsection{Selectivity of DNA hybridization recognition}

As a sensitive transduction technique, EIS has been widely used to investigate the interface property change of electrode surface. ${ }^{25}$ The selectivity of this DNA biosensor was investigated by using the probe ssDNA to hybridize with different DNA sequences of the PIK3CA gene related to gastric carcinoma. After the hybridization of the probe ssDNA, the changes of the Bode plots in $0.3 \mathrm{~mol} \mathrm{~L}^{-1} \mathrm{PBS}$ ( $\mathrm{pH} 7.0$ ) were shown in Fig. 4. Curve a was the

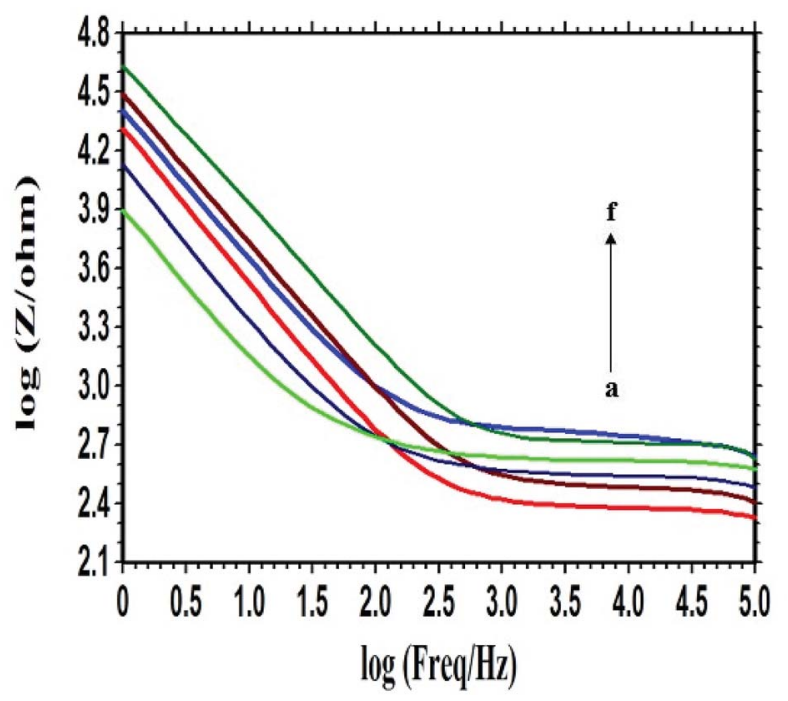

Fig. 4 Bode plots recorded at the PXa/WS $/$ /CPE (a), ssDNA/PXa/WS $/ 2$ CPE (f), dsDNA/PXa/WS 2 /CPE (hybridized with $C D N A$ ), (b), hybridized with ncDNA (e), hybridized with one-base mismatched DNA (c), and hybridized with two-base mismatched DNA (d) in $0.3 \mathrm{~mol} \mathrm{~L}^{-1} \mathrm{PBS}(\mathrm{pH} 7.0)$. 
Bode plot of the PXa/ $\mathrm{WS}_{2} / \mathrm{CPE}$. The impedance increased obviously when the probe ssDNA was attached onto the PXa/ $\mathrm{WS}_{2}$ nanocomposite (curve f), indicating the successful immobilization of the probe ssDNA. After the hybridization with the cDNA, a dramatic decrease of the impedance was observed (curve b). There are mainly two factors that may influence the change of impedance of the electrode after DNA hybridization. On one hand, it is well-known that dsDNA is more conductive than ssDNA. ${ }^{26,27}$ On the other hand, the DNA molecules can modulate the work function and the double layer thickness at the surface of the electrode due to the intrinsic negative charge of DNA molecules. $^{28}$ In this experiment, when the hybridization reaction occurs, an obvious decrease of impedance was observed, indicating that the first factor may occupy the chief status. And therefore the decrease of impedance after DNA hybridization in this experiment can be attributed to the much higher conductivity properties of dsDNA compared to ssDNA. When hybridized with the ncDNA, the impedance (curve e) changed little compared with the curve $\mathrm{f}$, illustrating that no hybridization occurred. The impedance related to the one-base mismatched DNA (curve c) and two-base mismatched DNA (curve d) were larger than the curve $b$, indicating that complete hybridization was not accomplished due to the base mismatch. The above results demonstrated that the indicator-free DNA biosensor based on the PXa/ $\mathrm{WS}_{2}$ nanocomposite had good selectivity for the DNA hybridization recognition.

\subsection{Quantitative analysis of complementary target sequences}

The probe ssDNA/PXa/ $\mathrm{WS}_{2} / \mathrm{CPE}$ was used to quantitatively detect the different concentrations of the complementary PIK3CA gene sequences, and the obtained Bode plots were shown in Fig. 5A. The difference (namely $\Delta \log Z$ ) between the $\log Z$ values of the probe-captured electrode and that after hybridization with the cDNA was adopted as the measurement signal. The results showed that the $\Delta \log Z$ value was linear with the logarithm of the PIK3CA gene target sequence concentrations in the range from $1.0 \times 10^{-15} \mathrm{~mol} \mathrm{~L}^{-1}$ to $1.0 \times$ $10^{-11} \mathrm{~mol} \mathrm{~L}^{-1}$ with the regression equation $\Delta \log Z=0.049 \log C$ +0.783 and the regression coefficient $(R) 0.9951$ (Fig. 5B). The detection limit could be calculated to be $1.6 \times 10^{-16} \mathrm{~mol} \mathrm{~L}^{-1}$ using $3 \sigma$ (where $\sigma$ was the relative standard deviation (RSD) of the blank solution, $n=11$ ). Compared with other reported works for the PIK3CA gene detection with the detection limit of $50 \mathrm{fM}$ and detection range (50 fM to $3200 \mathrm{fM}$ ) by localized surface plasmon resonance, ${ }^{29}$ and the detection limit of $10^{-17} \mathrm{M}$ and detection range $\left(10^{-16} \mathrm{M}\right.$ to $\left.10^{-13} \mathrm{M}\right)$ by differential pulse voltammetry, $^{30}$ these results showed that the developed indicator-free impedance detection assay in our work had a favorable sensitivity for electrochemical detection of DNA hybridization.

\subsection{Stability and reproducibility of the DNA biosensor}

The stability of the constructed DNA biosensor was tested. It was stored in the refrigerator at $4{ }^{\circ} \mathrm{C}$ for one week. No apparent change of EIS signals was observed after one week. In addition,
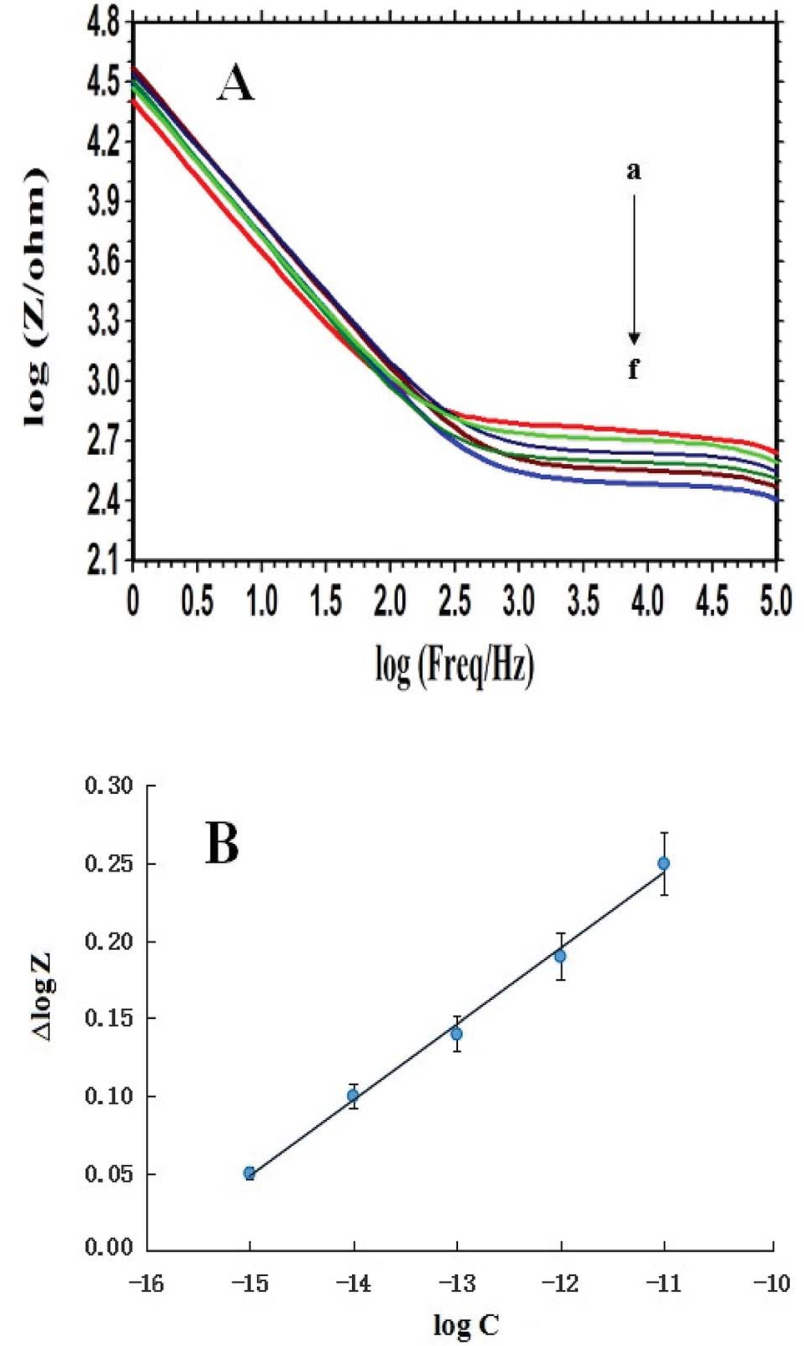

Fig. 5 (A) Bode plots recorded at the ssDNA/PXa/WS $2 / C P E$ (a) and after hybridization reaction with different concentrations of PIK3CA gene target sequences: (b) $1.0 \times 10^{-15}$, (c) $1.0 \times 10^{-14}$, (d) $1.0 \times 10^{-13}$, (e) $1.0 \times 10^{-12}$ and (f) $1.0 \times 10^{-11} \mathrm{~mol} \mathrm{~L}^{-1}$ in $0.3 \mathrm{~mol} \mathrm{~L}^{-1} \mathrm{PBS}(\mathrm{pH} 7.0$ ). (B) The plot of $\Delta \log Z$ versus the logarithm of the PIK3CA gene target sequence concentrations.

the probe-modified electrode was stored in PBS ( $\mathrm{pH} 7.0)$ at $4{ }^{\circ} \mathrm{C}$ for one month and the decrease of the EIS response was got as $5.68 \%$. The above results illustrated that the prepared DNA biosensor exhibited favorable stability and could be applied for further quantitative assay.

The reproducibility of this hybridization biosensor was measured by detecting $1.0 \times 10^{-15} \mathrm{~mol} \mathrm{~L}^{-1}$ target sequences with six parallel-made probe-captured electrodes. The results showed that a RSD of $5.8 \%$ for the signal changes was estimated, indicating that the biosensor had an acceptable reproducibility for DNA analysis.

\section{Conclusions}

In conclusion, a novel electrochemical sensing interface for detecting ctDNA related to gastric carcinoma was developed 
based on $\mathrm{WS}_{2}$ nanosheets supported PXa. The formed PXa/ $/ \mathrm{WS}_{2}$ nanocomposite possessed fine biocompatibility, as well as favorable electrochemical activity in a neutral environment. The self-signal changes of the PXa layer induced by DNA immobilization and hybridization could be sensitively recognized via $\mathrm{CV}$ and EIS. The total process is simple, direct and requiring no labelling processes or external indicators, and this procedure may be extremely useful for the cost effective mass production of a new generation of biosensors. The major limitations of the method lie in performing the assay with real blood samples. And we will further apply this sensor platform for the detection of specific DNA hybridization on real blood samples accompanied with the improvement of our experimental conditions.

\section{Conflicts of interest}

There are no conflicts to declare.

\section{Acknowledgements}

This work was supported by the Shandong Province Natural Science Foundation (No. ZR2017QB013) and the Undergraduate Training Program for Innovation and Entrepreneurship of Linyi University (No. 201810452170).

\section{References}

1 A. Rostami and S. V. Bratman, Utilizing circulating tumour DNA in radiation oncology, Radiother. Oncol., 2017, 124, 357-364.

2 X. Han, J. Y. Wang and Y. L. Sun, Circulating tumor DNA as biomarkers for cancer detection, Genomics, Proteomics Bioinf., 2017, 15, 59-72.

3 T. M. Butler, P. T. Spellman and J. Gray, Circulating-tumor DNA as an early detection and diagnostic tool, Curr. Opin. Genet. Dev., 2017, 42, 14-21.

4 L. Calapre, L. Warburton, M. Millward, M. Ziman and E. S. Gray, Circulating tumour DNA (ctDNA) as a liquid biopsy for melanoma, Cancer Lett., 2017, 404, 62-69.

5 E. Yong, Cancer biomarkers: written in blood, Nature, 2014, 511, 524-526.

6 W. Zhang, Z. C. Dai, X. Liu and J. M. Yang, High-performance electrochemical sensing of circulating tumor DNA in peripheral blood based on poly-xanthurenic acid functionalized $\mathrm{MoS}_{2}$ nanosheets, Biosens. Bioelectron., 2018, 105, 116-120.

7 G. Siravegna and A. Bardelli, Blood circulating tumor DNA for non-invasive genotyping of colon cancer patients, Mol. Oncol., 2016, 10, 475-480.

8 Z. Zou, P. Qi, Z. H. Qing, J. Zheng, S. Yang, W. J. Chen and R. H. Yang, Technologies for analysis of circulating tumour DNA: progress and promise, Trends Anal. Chem., 2017, 97, 36-49.

9 J. Das, I. Ivanov, E. H. Sargent and S. O. Kelley, DNA clutch probes for circulating tumor DNA analysis, J. Am. Chem. Soc., 2016, 138, 11009-11016.
10 A. Sajedi-Moghaddam, E. Saievar-Iranizad and M. Pumera, Two-dimensional transition metal dichalcogenide/ conducting polymer composites: synthesis and applications, Nanoscale, 2017, 9, 8052-8065.

11 Y. L. Hu, Y. Huang, C. L. Tan, X. Zhang, Q. P. Lu, M. Sindoro, X. Huang, W. Huang, L. H. Wang and H. Zhang, Twodimensional transition metal dichalcogenide nanomaterials for biosensing applications, Mater. Chem. Front., 2017, 1, 24-36.

12 Z. H. Hu, Z. T. Wu, C. Han, J. He, Z. H. Ni and W. Chen, Twodimensional transition metal dichalcogenides: interface and defect engineering, Chem. Soc. Rev., 2018, 47, 3100-3128.

13 A. Eftekhari, Tungsten dichalcogenides $\left(\mathrm{WS}_{2}, \mathrm{WSe}_{2}\right.$, and $\mathrm{WTe}_{2}$ ): materials chemistry and applications, J. Mater. Chem. A, 2017, 5, 18299-18325.

14 C. Lu, Y. B. Liu, Y. B. Ying and J. W. Liu, Comparison of $\mathrm{MoS}_{2}, \mathrm{WS}_{2}$, and graphene oxide for DNA adsorption and sensing, Langmuir, 2017, 33, 630-637.

15 Y. J. Chen, C. H. Kang, R. H. Wang, Z. Y. Ren, H. Y. Fu, Y. T. Xiao and G. H. Tian, $\mathrm{CoP} / \mathrm{WS}_{2}$ nanoflake heterostructures as efficient electrocatalysts for significant improvement in hydrogen evolution activity, Appl. Surf. Sci., 2018, 442, 352-360.

16 J. Ren, Z. Wang, F. Yang, R. P. Ren and Y. K. Lv, Freestanding $3 \mathrm{D}$ single-wall carbon nanotubes/ $\mathrm{WS}_{2}$ nanosheets foams as ultra-long-life anodes for rechargeable lithium ion batteries, Electrochim. Acta, 2018, 267, 133-140.

17 X. Shang, J. Q. Chi, S. S. Lu, J. X. Gou, B. Dong, X. Li, Y. R. Liu, K. L. Yan, Y. M. Chai and C. G. Liu, Carbon fiber cloth supported interwoven $\mathrm{WS}_{2}$ nanosplates with highly enhanced performances for supercapacitors, Appl. Surf. Sci., 2017, 392, 708-714.

18 K. C. Lin, Y. S. Li and S. M. Chen, Electrochemical determination of nicotinamide adenine dinucleotide and hydrogen peroxide based on poly(xanthurenic acid), flavin adenine dinucleotide and functionalized multi-walled carbon nanotubes, Sens. Actuators, B, 2013, 184, 212-219.

19 T. Yang, Q. Q. Kong, Q. H. Li, X. X. Wang, L. H. Chen and K. Jiao, Highly sensitive and synergistic detection of guanine and adenine based on poly(xanthurenic acid)reduced graphene oxide interface, ACS Appl. Mater. Interfaces, 2014, 6, 11032-11037.

20 F. A. S. Silva, C. B. Lopes, E. O. Costa, P. R. Lima, L. T. Kubota and M. O. F. Goulart, Poly-xanthurenic acid as an efficient mediator for the electrocatalytic oxidation of $\mathrm{NADH}$, Electrochem. Commun., 2010, 12, 450-454.

21 Y. W. Hu, T. Yang, X. X. Wang and K. Jiao, Highly sensitive indicator-free impedance sensing of DNA hybridization based on $\operatorname{poly}(m$-aminobenzenesulfonic $\mathrm{acid}) / \mathrm{TiO}_{2}$ nanosheet membranes with pulse potentiostatic method preparation, Chem. - Eur. J., 2010, 16, 1992-1999.

22 Q. D. Zhang, B. Piro, V. Noel, S. Reisberg and M. C. Pham, Functionalization of single-walled carbon nanotubes for direct and selective electrochemical detection of DNA, Analyst, 2011, 136, 1023-1028.

23 M. C. Pham, B. Piro and L. D. Tran, Direct electrochemical detection of oligonucleotide hybridization on poly(5- 
hydroxy-1,4-naphthoquinone-co-5-hydroxy-3-thioacetic acid1,4-naphthoquinone) film, Anal. Chem., 2003, 75, 6748-6752.

$24 \mathrm{~W}$. Zhang, Electrochemically reduced graphene oxide supported poly(indole-5-carboxylic acid) nanocomposite for genosensing application, $R S C$ Adv., 2015, 5, 103649-103655.

$25 \mathrm{~A}$. Bonanni and $\mathrm{M}$. del Valle, Use of nanomaterials for impedimetric DNA sensors: a review, Anal. Chim. Acta, 2010, 678, 7-17.

26 T. Y. Lee and Y. B. Shim, Direct DNA hybridization detection based on the oligonucleotide-functionalized conductive polymer, Anal. Chem., 2001, 73, 5629-5632.

27 H. R. Gu, X. Su and K. P. Loh, Electrochemical impedance sensing of DNA hybridization on conducting polymer filmmodified diamond, J. Phys. Chem. B, 2005, 109, 13611-13618.
28 F. Yan, S. M. Mok, J. J. Yu, H. L. W. Chan and M. Yang, Labelfree DNA sensor based on organic thin film transistors, Biosens. Bioelectron., 2009, 24, 1241-1245.

29 A. H. Nguyen and S. J. Sim, Nanoplasmonic biosensor: detection and amplification of dual bio-signatures of circulating tumor DNA, Biosens. Bioelectron., 2015, 67, 443449.

30 Y. L. Chu, B. Cai, Y. Ma, M. G. Zhao, Z. Z. Ye and J. Y. Huang, Highly sensitive electrochemical detection of circulating tumor DNA based on thin-layer $\mathrm{MoS}_{2} /$ graphene composites, RSC Adv., 2016, 6, 22673-22678. 